\title{
A Influência da Estruturação da Mensagem em Comportamentos Relacionados à Saúde: um teste experimental
}

\section{The Influence of Framing on Health-related Behaviors: an experimental test}

\author{
Verônica Feder Mayer \\ Doutora em Administração. Professora Adjunta da Universidade \\ Federal Fluminense. \\ Endereço: Rua Tiradentes, 171, Bloco 2, apto. 1203, CEP 24210-510, \\ Ingá, Niterói, Rio de Janeiro, RJ, Brasil. \\ E-mail: vmayerळgmail.com \\ Marcos Gonçalves Avila \\ Ph. D. in Accounting. Professor Associado II, Instituto Coppead \\ de Administração, UFRJ. \\ Endereço: Rua Pascoal Lemme, 355, Ilha do Fundão, CEP 21941-918, \\ Rio de Janeiro, RJ, Brasil. \\ E-mail: marcosळcoppead.ufrj.br
}

\section{Resumo}

Este estudo realizou um teste experimental envolvendo o efeito framing. Segundo a Teoria dos Prospectos, o efeito framing é a possibilidade de influenciar a decisão de um indivíduo sem distorcer a informação ou suprimi-la por meio de mudanças sutis na estruturação do problema. Foi testada a hipótese de que um folheto enfatizando as consequências negativas de não se prevenir contra o estresse seria mais persuasivo do que um folheto que enfatizasse as consequências positivas de se prevenir. Estudos anteriores, que examinaram o efeito framing na estruturação de mensagens persuasivas, produziram resultados variados. Esta pesquisa explorou as explicações teóricas para os resultados obtidos em campanhas relacionadas à saúde. Estudantes brasileiros de programas de pós-graduação foram expostos a um folheto estruturado negativamente, a um folheto estruturado positivamente ou a um folheto simplesmente informativo sobre o estresse. Atitudes em relação às recomendações para prevenir-se contra estresse e as intenções de seguilas foram medidas. Os resultados obtidos indicam maior persuasão do framing negativo.

Palavras-chave: Teoria dos prospectos; Efeito framing; Mensagens em saúde; Estresse. 


\section{Abstract}

This study performed an experimental test involving the framing effect. According to the Prospect Theory, the framing effect is the possibility of influencing an individual's decision without distorting the information or suppressing it, but by means of subtle changes in the way the problem is presented. We tested the hypothesis that a brochure emphasizing the negative consequences of not protecting oneself against stress would be more persuasive than a brochure emphasizing the positive consequences of this attitude. Prior studies, which examined the framing effect in structuring persuasive messages, have produced varied results. This study explored the theoretical explanations for results obtained in health campaigns. Brazilian postgraduate students were exposed to a negatively structured brochure, a positively structured brochure or a brochure that merely informed about stress. Attitudes concerning recommendations to protect oneself from stress and the intentions of following them were measured. The results indicate that the negative framing is more persuasive.

Keywords: Prospect Theory, Framing Effect, Health Messages, Stress

\section{Introdução}

A importância da mídia de massa na educação sobre riscos relacionados à saúde tem aumentado muito nos últimos anos, com campanhas de utilidade pública dedicadas a prevenção de Aids, abuso de álcool e drogas, acidentes nas estradas, a importância da amamentação e vacinação, a prevenção de certos tipos de câncer e doenças do coração, entre outras (Rothman e col., 2006; Chun-Tuan, 2007). Nos EUA, a mídia tornou-se a maior fonte de informações sobre riscos e comportamentos relacionados à saúde, segundo um estudo da American Cancer Society (Meyerowitz e Chaiken, 1987). A prevenção e a detecção de doenças, ainda em estágios nos quais a cura é possível, é um tema de extrema relevância para a medicina moderna. Estima-se que de 40\% a 70\% de todas as mortes prematuras e, aproximadamente, $66 \%$ das deficiências físicas poderiam ser prevenidas por meio do controle de menos do que dez fatores de risco, como dieta, exercícios e abuso de álcool (Signorelli, 1993). Cresce, portanto, a importância de se compreender os fatores que influenciam a eficácia dos apelos da mídia que visam a mudanças de comportamentos relacionados à saúde.

A Teoria dos Prospectos (Kahneman e Tversky, 1979; Tversky e Kahneman, 1981) trouxe novas possibilidades de estudos comportamentais em diversos campos. Em marketing foram abertas importantes linhas de pesquisa, como gerenciamento de preços e valor (Smith e Nagle, 1995; Serpa e Avila, 2004) e preferências e escolhas do consumidor (Simonson, 1993). Muitos desses estudos têm buscado determinar o impacto no comportamento do que Tversky e Kahneman (1981) denominaram de efeito framing, ou seja, a possibilidade de influenciar a decisão de um indivíduo sem distorcer a informação ou suprimi-la, por meio de mudanças sutis na sua estruturação.

Os termos framing positivo e framing negativo foram usados para descrever duas formas de apresentar a mesma informação, positiva ou negativamente (Tversky e Kahneman, 1981). No âmbito da saúde, estudos foram conduzidos na tentativa de se compreender os efeitos do framing positivo e negativo nas intenções de desempenhar comportamentos de prevenção e de detecção de doenças (Meyerowitz 
e Chaiken, 1987; Masheswaran e Meyers-Levy, 1990; Rothman e col., 1993; Block e Keller, 1995; Smith e Nagle, 1995; Chandran e Menon, 2004; Gifford e Bernard, 2006; Kim, 2006; Rothman e col., 2006; Chang, 2007; Chun-Tuan, 2007). Rothman e colaboradores (2006), por exemplo, verificaram que, para campanhas publicitárias que alertam sobre problemas de saúde, o framing positivo se mostra mais eficiente quando o objetivo é alertar sobre os fatores que podem impedir o aparecimento da doença e o framing negativo é mais efetivo para alertar como detectar a doença.

O objetivo deste trabalho é contribuir com o estudo do efeito framing e suas aplicações na comunicação de marketing, mais especificamente em campanhas relacionadas à saúde. Este artigo apresenta a Teoria dos Prospectos em conjunto com suas aplicações, e as contribuições posteriores. Apresenta pesquisa experimental feita com o objetivo de testar o efeito framing numa campanha de saúde junto a consumidores brasileiros. Este trabalho limita-se ao estudo do efeito framing na comunicação de marketing, segundo a linha proposta pela Teoria dos Prospectos e pelas pesquisas posteriores que ampliaram sua definição. Não são estudadas outras influências no comportamento dos consumidores.

\section{0 estresse no cenário atual}

Para este estudo foi escolhido o estresse, tema cuja relevância vem aumentando desde as transformações sociais e econômicas ocorridas a partir do final do Século XX (Araújo e col., 2003; Sparrenberger e col., 2003). Recentemente, a Organização Mundial da Saúde descreveu o estresse como a maior epidemia mundial deste século.

0 estresse é um fenômeno comum na era da informação. Nos Estados Unidos, a National Mental Health Association afirma que de $75 \%$ a $90 \%$ de todas as visitas a médicos estão relacionadas ao estresse: estima-se que o estresse no trabalho custe mais de U\$20o bilhões anuais para a indústria norteamericana, e que de $60 \%$ a $80 \%$ dos acidentes de trabalho nos Estados Unidos estejam relacionados ao estresse (Rosenfield, 200o).

Uma recente pesquisa realizada pelo Centro
Psicológico de Controle do Stress (CPCS), sediado em São Paulo, mostrou que no Brasil a incidência de estresse na população adulta é de 32\% (Lipp, 2007). Esse percentual pode ser confrontado com os dados da International Stress Management Association (ISMA), segundo os quais $70 \%$ dos brasileiros economicamente ativos sofrem de estresse, fazendo com que os brasileiros ocupem o segundo lugar no ranking dos povos mais estressados do mundo, perdendo apenas para os japoneses.

$\mathrm{O}$ aumento de responsabilidade, bem como a exigência de autocontrole nas atividades profissionais, ocasiona o aumento do nível de estresse na esfera organizacional e, por conseguinte, na sociedade brasileira (Araújo e col., 2003; Palácios e col., 2002). Sparrenberg e colaboradores (2004) apontam como causas muito frequente de estresse a separação conjugal, a instabilidade socioeconômica - em especial o desemprego - e as doenças familiares.

As condições de trabalho na contemporaneidade parecem não permitir que se viva em nível tolerável de estresse (Bastos e col., 2002): o crescente avanço tecnológico, as exigências de capacitação profissional e de competitividade empresarial são alguns dos fatores que aumentam as doenças psíquicas e físicas as quais estão expostos os trabalhadores.

0 estresse revela-se como um processo socialmente construído (Pereira e col., 20o6), e para entendê-lo no ambiente de trabalho é necessário estudar a relação entre trabalho e vida pessoal. Em organizações públicas, fatores emocionais e fatores sociais presentes no ambiente de trabalho foram apontados como principais causas do estresse ocupacional psicológico (Balassiano e col., 2005).

As pesquisas também mostram que o estresse não é uma doença dos mais velhos. Em maior ou menor grau, os jovens sentem aumentar a pressão do ambiente e sofrem cada vez mais de estresse. A ruptura do bem-estar individual causada pela incapacidade de se superar a vivência de experiências estressantes é chamada de distress. No Brasil, as mulheres, os mais velhos, os mais pobres e os indivíduos de menor escolaridade são os grupos que apresentam níveis mais altos de distress (Sparrenberger e col., 2003). 


\section{Julgamento e Tomada de Decisão: a teoria dos prospectos}

Tversky e Kahneman (1981) propõem que explicações e predições sobre as escolhas das pessoas são frequentemente fundadas na presunção da racionalidade humana. A decisão racional requer que problemas que tenham os mesmos elementos básicos - alternativas de ação, resultados e probabilidades - levem à mesma decisão; e que as preferências não sejam afetadas por variações na maneira como os elementos básicos da decisão são apresentados. Entretanto, por causa das limitações da percepção e das estratégias subjetivas de julgamento, isso nem sempre é verdade. Como a Teoria dos Prospectos pretende descrever o comportamento, ela incorpora e descreve os desvios da racionalidade, que resultam de processos automáticos de decisão.

Segundo a Teoria dos Prospectos, o indivíduo avalia os resultados de uma decisão como ganhos ou perdas percebidos em relação a um ponto de referência fixo e neutro, que é adotado para a decisão em questão. Esse ponto de referência pode ser o status $q u o$, nível de aspirações do indivíduo ou ser sugerido pela maneira como o problema é estruturado. A cada resultado é associada ainda uma medida de atratividade e satisfação para o tomador de decisão, que os autores denominam de valor. A percepção de valor é regida pela função valor (Figura 1), côncava para ganhos e convexa para perdas, sendo mais acentuada nas perdas do que nos ganhos. $\mathrm{O}$ valor percebido das consequências da escolha, sendo positivas ou negativas, tem características de retornos decrescentes. A diferença, por exemplo, entre R\$ 10,00 e R\$ 20,00 parece maior do que a diferença entre R\$ 110,00 e R\$ 120,oo, embora em termos absolutos as quantias sejam idênticas. A função valor também indica que a reação à perda é mais extrema do que a resposta ao ganho; em outras palavras, a dor associada à perda de uma determinada quantia é maior do que o prazer associado ao ganho da mesma quantia.

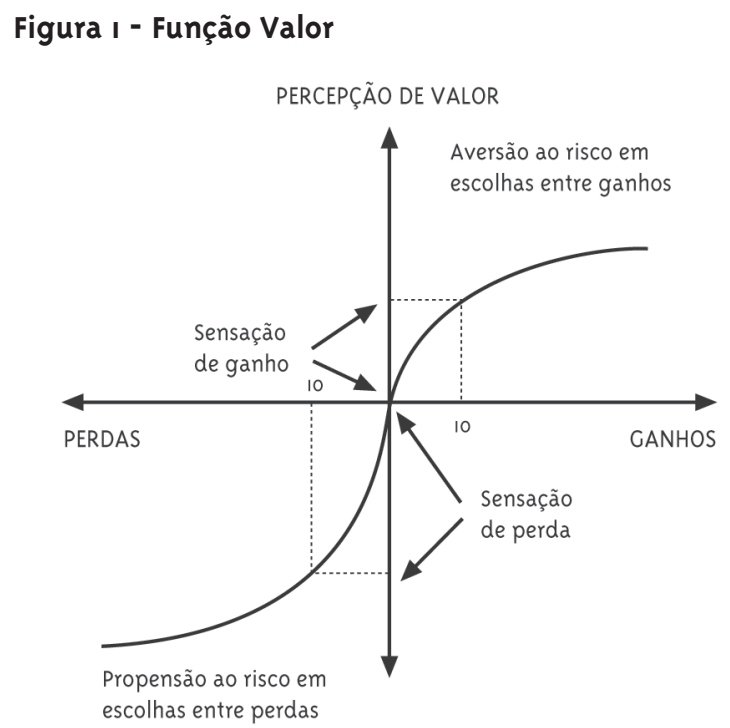

Fonte: Tversky e Kahneman (1984, p. 342).

A função valor sugere ainda que os indivíduos tratam os riscos relativos a ganhos percebidos de forma diferente dos riscos que dizem respeito a perdas percebidas. Assim, escolhas entre opções envolvendo resultados localizados na região de ganhos são, em geral, avessas a riscos e escolhas entre opções envolvendo perdas são, em geral, propensas ao risco.

Como a estruturação de um problema envolve a sugestão de um ponto de referência em particular, a forma como um problema é apresentado pode alterar o ponto neutro percebido e, em consequência, alterar a percepção em relação aos resultados da decisão de ganhos para perdas ou vice-versa. Essa alteração na percepção dos resultados, por sua vez, influencia a postura em relação a risco por parte do tomador de decisão e pode levar a uma reversão de preferências sobre as alternativas disponíveis, caracterizando o efeito framing.

\section{0 efeito framing}

O efeito framing constitui-se na possibilidade de influenciar a decisão de um indivíduo sem distorcer a informação ou suprimi-la por meio de mudanças sutis na apresentação e estruturação das informações de um mesmo problema. A avaliação de resultados é suscetível ao efeito framing por conta 
da não linearidade da função valor, por causa da tendência das pessoas de avaliar opções em relação ao ponto de referência, que pode ser sugerido ou implícito pelo enunciado do problema, e pela postura diferenciada em relação ao risco dependendo da região da função valor em que os resultados são percebidos - ganhos ou perdas. Em um conjunto de experimentos, os autores obtiveram mudanças sistemáticas de preferências por meio da variação na estruturação e apresentação de opções, resultados e contingências associados a uma escolha particular (Tversky e Kahneman, 1981). Os resultados da pesquisa empírica desenvolvida pelos autores têm sido replicados com diferentes formulações de questões decisórias (Avila e Sá, 1996; Bazerman, 2006; Serpa e Avila, 2004).

\section{Efeito Framing em Mensagens Relacionadas à Saúde}

Pesquisadores das áreas de psicologia e julgamento têm afirmado que as pessoas respondem de maneira diferente a uma ideia quando ela é apresentada com framing positivo ou negativo (Rothman e col., 1993). Uma vez que maior importância é dada à avaliação negativa do que à avaliação positiva de um dado objeto, o framing negativo de uma mensagem é considerado mais efetivo que o framing positivo (Matos e Veiga, 2004). Entretanto, o framing de mensagem nem sempre produziu efeitos consistentes no comportamento em geral, e no comportamento relacionado à saúde, em particular.

Por meio de seu experimento sobre autoexame para prevenção do câncer de mama, Meyerowitz e Chaiken (1987) mostraram que as mulheres que leram o folheto contendo o framing negativo (perdas por não fazer o exame) manifestaram mais atitudes positivas, intenções e comportamentos relacionados ao exame, do que as que leram o folheto contendo o framing positivo (ganhos fazendo o exame) e do que as que leram folhetos sem argumentos persuasivos.

Recentemente, realizando um estudo sobre efeito framing na compra de alimentos orgânicos, Gifford e Bernard (2006) mostraram que enfatizar os benefícios de consumir esses produtos é mais efetivo do que enunciar os males causados pelos produtos da agricultura convencional. Estudando o efeito framing para anúncios de produtos de saúde para uso pessoal, Chun-Tuan (2007) mostrou que anúncios para produtos familiares são sempre mais efetivos quando enfatizam as vantagens que a compra do produto trará e que utilizar framing positivo e negativo na mesma mensagem só traz resultados eficientes se o consumidor tiver experiência prévia no assunto.

Maheswaran e Meyers-Levy (1990) realizaram um estudo sobre como as atitudes e os comportamentos em relação a uma campanha de prevenção de doenças do coração são afetados pelo framing de mensagem, em situações de alto e baixo envolvimento. Por meio da condução de um experimento, os autores encontraram relação entre o tipo de framing usado e o nível de envolvimento da audiência com o assunto da mensagem. Em situação de alto envolvimento, o framing negativo foi mais efetivo e mais persuasivo, e em situação de baixo envolvimento funcionou melhor o framing positivo. Os mesmos pesquisadores verificaram que quando o envolvimento era baixo, os indivíduos abstiveramse de processar a mensagem em detalhe e basearam suas atitudes em simples deduções. Pelo fato de, sob baixo envolvimento, as pessoas construírem suas deduções a partir de inferências com as quais concordam, a mensagem foi mais persuasiva quando estruturada de forma positiva.

No que se diz respeito ao efeito framing em mensagens relacionadas à saúde, quando a pessoa está mal-humorada o framing positivo é mais eficiente; em contrapartida, quando ela está bem-humorada, é mais efetivo o framing negativo (Chang, 2007). Ao comparar diferentes estratégias de comunicação para produtos relacionados à saúde, mais especificamente medicamentos para emagrecimento, Huertas e Campomar (2006) mostraram que mensagens com apelos predominantemente racionais obtiveram atitudes e intenções comportamentais mais favoráveis por parte dos consumidores brasileiros.

O estudo de Block e Keller (1995) testou o efeito da eficácia percebida ${ }^{1}$ e do framing da mensagem sobre intenções de adoção de um comportamento

1 Eficácia percebida é o grau de certeza que um indivíduo percebe sobre um remédio ou tipo de tratamento. 
relacionado à saúde. Os resultados indicaram que quando não está claro que ao se seguir determinada recomendação se chegará aos resultados desejados (condição de baixa eficácia), um maior e mais profundo processamento de informações é motivado. Além disso, no contexto de um maior e mais profundo processamento de informações, o framing negativo é mais persuasivo do que o framing positivo. Em contraste, na condição de alta eficácia, foi observado um menor esforço de processamento da mensagem e os dois tipos de framing - positivo e negativo - se mostraram igualmente persuasivos.

Cabe ressaltar que o efeito framing em mensagens relacionadas à saúde não acontece somente em função da abordagem negativa ou positiva de um mesmo assunto. O framing temporal também influencia a percepção de risco, sendo mais efetivo apresentar estatísticas em número de casos por dia do que por ano (Chandran e Menon, 2004).

A Protection Motivation Theory é responsável por incorporar o papel da eficácia percebida na comunicação de saúde. 0 modelo assume que receber uma comunicação sobre saúde faz com que as pessoas avaliem (1) a gravidade do evento, (2) a probabilidade de o evento ocorrer e (3) sua crença na eficácia das recomendações. 0 mais importante previsor de intenções é a eficácia das recomendações em levar ao resultado desejado, o que significa que a adoção tende a ocorrer principalmente quando a pessoa sente que o comportamento recomendado tem grande probabilidade de levar ao resultado desejado (Tanner e col., 1991).

\section{0 papel do envolvimento}

Envolvimento 2 é definido por Kardes (2002) como a relevância ou importância percebida de determinada questão. Uma pessoa altamente envolvida com um tema ou decisão tem maior chance de pensar mais sobre o assunto ou de ter sentimentos mais intensos (Bochner e Insko, 1966).

Tido por alguns autores como o determinante primário do comportamento do consumidor (Jeunon, 2005), o envolvimento denota a intensidade com a qual um produto está imbuído nos sistemas de valo- res dos consumidores, sendo a principal variável que explica as diferenças no grau em que um indivíduo está disposto a realizar esforços mentais e físicos para cumprir atividades relacionadas com o consumo. O nível de envolvimento do consumidor pode ser tratado como um reflexo de quanto o indivíduo investiu financeiramente e/ou emocionalmente no processo de consumo (Freire e Nique, 2005).

Modelos de persuasão encontrados na literatura do marketing sugerem que pessoas que estão altamente envolvidas com uma questão têm maior probabilidade de processar mensagens relevantes em detalhe. Sob alto envolvimento, a maneira como as pessoas combinam ou integram a mensagem relevante numa atitude única tem um importante papel na persuasão (Petty e col., 1983). Durante a integração, a informação negativa com frequência recebe maior peso e influência do que a informação positiva. A implicação disso é que, se o envolvimento com a questão for alto, mensagens relevantes não apenas serão processadas em detalhe, mas também devem ser mais persuasivas quando forem estruturadas negativamente.

No estudo de Maheswaran e Meyers-Levy (1990) o framing positivo foi mais efetivo do que o negativo quando os indivíduos não estavam motivados a processar a mensagem detalhadamente, ou seja, quando o envolvimento era baixo - o que, em geral, acontece com campanhas de massa relacionadas à saúde. Com base nos estudos anteriormente realizados, é possível aventar que, para o tema saúde, em situações nas quais o nível de eficácia for baixo e o envolvimento do público for alto, ou seja, quando houver maior processamento da informação, o framing negativo deve ser mais efetivo do que o positivo.

\section{Hipóteses}

Em consonância com as pesquisas conduzidas anteriormente (Maheswaran e Meyers-Levy, 1990; Block e Keller, 1995; Matos e Veiga, 2004; Chang, 2007), e em acordo com a Teoria dos Prospectos, este estudo prevê que o framing negativo deve ter mais impacto em intenções e atitudes do que o framing

2 Apesar de numerosos fatores influenciarem o envolvimento da audiência, alguns importantes são a natureza da categoria do produto caracterizado no anúncio, a mídia selecionada para comunicar a mensagem, e o veículo escolhido em particular (Maheswaran e MeyersLevy, 1990). 
positivo, em um folheto de prevenção do estresse, em que haja alto envolvimento do participante.

As hipóteses de pesquisa, em suas formas nula e alternativa, são descritas a seguir:

$\mathrm{H}_{\mathrm{o} 1}$ : A inclusão do texto persuasivo (objeto de manipulação de framing) não terá influência na atitude e nas intenções de seguir o comportamento recomendado.

$\mathrm{H}_{11}$ : A inclusão do texto persuasivo (objeto de manipulação de framing) terá influência na atitude e nas intenções de seguir o comportamento recomendado.

$\mathrm{H}_{\mathrm{o} 2}$ : A estruturação da mensagem, ou framing, não terá influência na atitude e nas intenções de seguir o comportamento recomendado.

$\mathrm{H}_{12}$ : Mensagens estruturadas negativamente levarão a atitudes mais favoráveis e a maiores intenções de seguir o comportamento recomendado do que mensagens estruturadas positivamente.

\section{Metodologia}

O trabalho teve um caráter empírico, investigando o comportamento de pessoas por meio de um experimento, em que foram aplicados questionários que simularam um folheto sobre prevenção do estresse.

A fim de descrever a influência de diferentes estruturações de uma mesma mensagem, este estudo trabalhou com uma variável independente: tipo de mensagem (negativa ou positiva). A variável dependente foram atitudes e intenções em relação ao comportamento recomendado. Como a hipótese visava testar o efeito framing, o experimento foi composto de um conjunto de textos e de um questionário, em três diferentes versões, por meio de uma metodologia consistente com o experimento realizado por Meyerowitz e Chaiken (1987):

\section{Versão 1 - Negativa}

Página 1: apresentação do experimento, instruções e questionário de levantamento de dados; página 2: texto informativo sobre estresse; Página 3: texto com framing negativo; página 4: recomendações; página 5: frase de reforço do framing negativo; página 6: questionário.

\section{Versão 2 - Positiva}

Página 1: apresentação do experimento, instruções e questionário de levantamento de dados; página 2: texto informativo sobre estresse; página 3: texto com framing positivo; página 4: recomendações; página 5: frase de reforço do framing positivo; página 6: questionário.

\section{Versão 3 (grupo de controle) - Sem manipulação de framing}

Página 1: apresentação do experimento, instruções e questionário de levantamento de dados; página 2: texto informativo sobre estresse; página 3: recomendações; Página 4: questionário.

Um total de 120 estudantes brasileiros de programas de pós-graduação de uma instituição privada de ensino superior, que estudavam à noite e trabalhavam durante o dia, de ambos os sexos, concordaram em participar voluntariamente da pesquisa. Esses estudantes sofrem uma grande pressão em relação ao seu desempenho, tanto na empresa, quanto no curso, utilizando poucas horas da sua rotina para o lazer. Isso faz com que estejam muito expostos ao risco de sofrer de estresse. $\mathrm{O}$ objetivo foi escolher uma amostra relativamente homogênea de pessoas.

Cada versão do questionário foi aplicada a 40 respondentes, no verão de 2001. A amostra resultante dos 120 questionários aplicados foram 104 questionários válidos. Antes da aplicação do questionário final, diversos pré-testes foram realizados, com alunos da mesma instituição e profissionais de empresas, envolvendo 6 o pessoas durante dois meses. Os resultados desses testes levaram a diversos refinamentos no desenho do experimento.

\section{Variável independente}

A manipulação do efeito framing nesse experimento seguiu o padrão usado pelos autores dos experimentos anteriores: a mensagem enfatiza consequências positivas envolvidas na adoção das recomendações ou consequências negativas envolvidas na não adoção das recomendações. Exemplo da manipulação do framing neste estudo, em que: [positivo] (negativo)

Portanto, se você (não) aprender a observar seu organismo e a se prevenir dos perigos do estresse, aumentará (diminuirá) suas chances de crescer em sua carreira profissional e de ter sucesso no campo pessoal. Quem (não) previne e (não) controla o estresse, (reduz) [eleva] a qualidade de vida e (diminui) [aumenta] sua produtividade no trabalho. 


\section{Variável dependente}

Segundo Aaker e colaboradores (2001), atitudes são estados mentais usados pelas pessoas para estruturar a maneira como percebem seu ambiente, bem como para guiar a forma como respondem a ele. Existe uma aceitação geral de que existem três componentes relacionados que formam as atitudes: (1) cognição ou conhecimento; (2) afeição ou gostar; e (3) intenção ou ação. 0 componente cognição representa a informação que uma pessoa detém sobre um objeto e inclui ciência da existência do objeto, crenças sobre suas características e julgamentos sobre a importância dessas características. O componente afetivo resume os sentimentos em relação ao objeto. 0 componente intenção refere-se às expectativas futuras de comportamento em relação ao objeto.

A variável dependente foi medida, no total, por meio de quatro perguntas, cujas respostas foram somadas, para a obtenção de um índice de atitudes/ intenções: uma pergunta para medição do julgamento dos respondentes sobre a importância das recomendações, e mais três perguntas para medição da intenção de adotar o comportamento recomendado no texto: Você tem intenção de seguir as recomendações? Qual é a chance de que você passe a adotar pelo menos uma recomendação este ano? Quantas recomendações, se alguma, você pretenderia adotar no decorrer deste ano?.

Foi usada uma escala Likert de cinco pontos para todas as perguntas ligadas à variável dependente. Uma suposição desse método de escala é que cada um dos itens mede um aspecto de um fator comum (atitudes/intenções), sendo possível legitimar a soma dos resultados. 0 uso da escala Likert é consagrado nesse tipo de pesquisa (Aaker e col., 2001).

\section{Questionário, texto informativo sobre estresse $e$ recomendações}

Foi elaborado um questionário de levantamento de dados apresentado antes da leitura dos textos. Seu primeiro objetivo foi identificar a faixa etária e o sexo dos respondentes. O segundo objetivo foi verificar o conhecimento anterior e o envolvimento potencial do indivíduo com o tema do experimento. Consistente com Block e Keller (1995), esse mecanismo proporciona um perfil genérico do pesquisado e possibilita identificar algum tipo de interação exis- tente entre o conhecimento anterior e as respostas do questionário principal.

A elaboração do texto informativo e das recomendações usadas no experimento contou com a colaboração da equipe técnica do portal de saúde da internet Planeta Vida. A grande preocupação foi manter as informações livres de conotações muito negativas ou muito positivas, o que em parte foi resolvido por meio do teor altamente informativo do texto, sem muitos argumentos persuasivos, em que tanto as características positivas como as negativas do estresse foram relatadas.

\section{Checagens}

Para avaliar a atitude dos respondentes em relação ao conjunto de textos, (credibilidade, utilidade e clareza) foi aplicada uma escala de diferencial semântico de cinco pontos. A escala usada no experimento foi baseada na escala desenvolvida e testada por MacKenzie e Lutz (1989) para medição de atitudes em relação à propaganda em geral. As escalas de diferencial semântico têm sido muito usadas em pesquisa de marketing para se avaliar a atitude em relação a anúncios e a outros instrumentos de comunicação (Bruner e Hensel, 1996). Para saber se houve percepção de diferença de framing entre as versões do questionário, foi formulada uma questão, utilizando uma escala tipo Likert de cinco pontos, na qual o entrevistado foi instruído a marcar se o texto enfatizou os ganhos para a saúde que a prevenção do estresse pode trazer ou as perdas para a saúde que a falta de prevenção do estresse pode trazer.

Envolvimento é uma variável relevante no que se refere a suposições feitas para a formulação da hipótese. A escolha dos participantes foi proposital, no sentido de obter um cenário de alto envolvimento. 0 grau de relevância do tema, que reflete o envolvimento, foi verificado de duas maneiras. No questionário inicial, perguntamos aos entrevistados se eles ou alguém próximo a eles já havia sido diagnosticado por um médico como sofrendo de estresse. No questionário, foi incluída uma pergunta a que o respondente deveria marcar qual o grau de risco de uma pessoa como ele vir a sofrer de estresse. Novamente foi usada uma escala Likert de cinco pontos. A importância percebida do assunto e as atitudes em relação ao texto também foram indicadores do grau de envolvimento. 


\section{Tratamento dos dados}

Os dados foram tratados com o uso de dois métodos. Inicialmente foi através do teste Anova (Winer e col., 1991). A análise de variância é uma extensão do teste t para dois ou mais grupos. Esse teste examina a variabilidade entre as médias das amostras em relação à difusão das observações dentro de cada grupo. A hipótese nula é que as amostras dos valores vêm de populações com médias iguais. Posteriormente, foi aplicado o teste t para a análise de múltiplas comparações, sendo realizada a comparação das médias de cada uma das versões dos questionários, duas a duas. Esse método é comumente aplicado a situações nas quais há necessidade de comparar as médias de dois grupos, nos quais os indivíduos foram aleatoriamente incluídos, e as diferenças nas respostas são, então, apontadas como efeito da manipulação de variáveis dependentes (Ragano, 1986). Foi adotado o nível de significância de 10\%, para rejeitar a hipótese nula.

\section{Resultados}

\section{Teste das hipóteses}

Os resultados da variável dependente foram obtidos por meio do somatório de quatro perguntas, gerando um índice de atitudes/intenções consistente com procedimento normalmente adotado por meio da escala de Likert (ver Aaker e col., 2001). No gráfico que aparece adiante estão representadas as médias de atitudes/intenções das três versões dos questionários. 0 teste Anova, que compara as médias dos três grupos, teve como resultado $(F=4,950$; sig. = $o, 008$ ), que indicou existirem diferenças significativas entre elas. No entanto, o Anova não é suficiente para sabermos se os questionários com manipulação de framing têm resultados melhores do que os questionários do grupo de controle. Também não é suficiente para afirmarmos qual das versões (positiva, negativa ou controle) está influenciando mais as atitudes e intenções. Por esse motivo, foi realizado o teste t para a análise de comparações múltiplas, com a comparação das médias de cada uma das versões dos questionários, duas a duas.

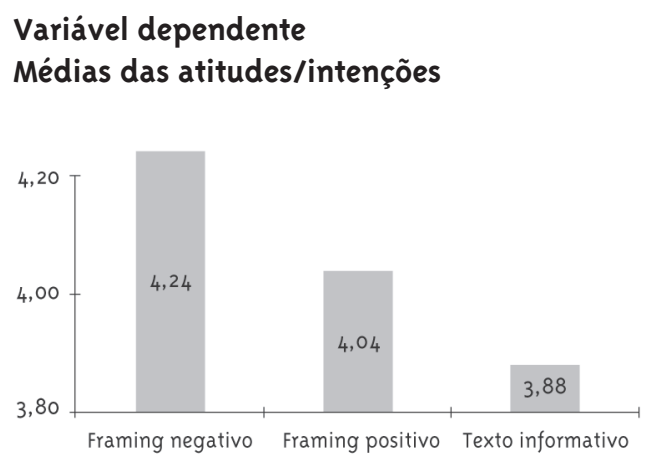

A primeira hipótese deste experimento dizia respeito à inclusão de um texto com manipulação de framing e de como ele deveria afetar atitudes e intenções. O teste t que compara as versões negativas e controle dos questionários indica que houve diferença significativa entre as respostas dos questionários estruturados negativamente e dos questionários apenas informativos, em favor do framing negativo $(F=15,299 ;$ sig. = o,ooo $)$. Não se pode afirmar, porém, que houve diferença significativa entre as versões positiva e controle dos questionários ( $F=2,294$; sig. $=0,131$ ), apesar de a média do questionário positivo ser um pouco maior do que a do questionário de controle. Esses resultados conirmam os efeitos do texto negativo na persuasão. Portanto, $\mathrm{H}_{\mathrm{o} 1}$ é rejeitada, pois a inclusão do texto com framing teve maior efeito nas atitudes e intenções de seguir o comportamento recomendado.

A segunda hipótese deste experimento dizia respeito à forma da estruturação do problema e de como ela deveria afetar atitudes e intenções. 0 teste t que compara as versões negativa e positiva dos questionários $\left(F=5,299\right.$; sig. = o, 022) indica que $\mathrm{H}_{02}$ é rejeitada em favor de $\mathrm{H}_{12}$, ou seja, houve diferença significativa entre as respostas dos questionários estruturados negativamente e dos questionários estruturados positivamente, em favor do framing negativo. Atitudes mis favoráveis e maiores intenções de seguir o comportamento recomendado foram encontrados na versão negativa, conforme a previsão feita na hipótese.

\section{Checagens}

Segundo os resultados obtidos, os indivíduos que responderam às três diferentes versões do questionário apresentam a mesma distribuição em termos 
de sexo e de idade. 0 mesmo acontece com a intimidade das pessoas em relação ao tema, quando relatam se já houve diagnóstico de estresse envolvendo elas mesmas ou pessoas próximas. Dessa forma, podemos descartar a possibilidade de ter ocorrido interferência nas respostas finais em decorrência de diferentes perfis dos indivíduos. Os respondentes deste experimento foram principalmente homens (71\%), divididos em dois grandes grupos etários, de 20 a 29 anos (41\%) e de 30 a 39 anos (42\%).

O conjunto de textos do experimento, que simulou o folheto de prevenção do estresse, foi avaliado pelos respondentes e os resultados apontaram um desempenho muito favorável. A escala de diferencial semântico utilizada testou a percepção de credibilidade, utilidade e clareza (inteligibilidade). Não houve diferença significativa entre as três versões do questionário nessa questão. A média alta obtida (média total = 4,153; escala de 1 a 5) reflete aceitação, credibilidade e compreensão do conjunto de textos muito boas.

Em relação ao aspecto envolvimento, a relevância do tema para os respondentes está presente, em primeiro lugar, no resultado de $68 \%$ obtido na questão que investigou se houve diagnóstico de estresse envolvendo eles mesmos ou pessoas próximas. Em segundo lugar, quando apenas $27 \%$ dos participantes do experimento consideraram ter um risco baixo ou muito baixo de sofrer de estresse. Em terceiro lugar, quando a maioria (86\%) declarou considerar as recomendações do folheto importantes ou muito importantes. De acordo com esses dados, verificamos que foi atingido nosso objetivo de alcançar um grupo com alto envolvimento com o tema.

A checagem do framing foi bem-sucedida. As médias (negativo $=3,294 ;$ positivo $=2,571$; controle $=$ $3,0 o o)$ e o teste Anova $(F=2,382$; sig. $=o, 098)$ indicaram que houve percepção diferenciada entre as versões dos questionários.

\section{Conclusões}

A hipótese de que a informação negativa tem maior impacto do que a informação positiva equivalente é consistente com as propriedades da função valor da Teoria dos Prospectos, ou seja, a intensidade da sensação no domínio da perda é maior do que a in- tensidade da sensação no domínio do ganho. Assim, sem suposições adicionais, seria possível prever que um folheto enfatizando os aspectos negativos de não se prevenir contra o estresse teria maior impacto persuasivo do que um folheto enfatizando aspectos positivos de se prevenir contra o estresse.

No campo da persuasão, Petty e colaboradores (1983) encontraram evidências de que o processo de persuasão é diferente quando os consumidores processam mais profundamente a mensagem. A rota central para a persuasão é mais adequada em situações de alto envolvimento, quando o consumidor presta atenção aos argumentos principais, reflete sobre eles e fortalece ou muda sua atitude com relação ao assunto. Sob alto envolvimento, a informação negativa, com frequência, recebe maior peso e influência do que a informação positiva (Matos e Veiga, 2004). A rota periférica para a persuasão é mais adequada em situações de baixo envolvimento, quando o consumidor não presta atenção na mensagem, apenas no cenário, na trilha sonora e nos títulos. Portanto, em campanhas nas quais seja possível criar condições de alto envolvimento, havendo maior processamento da informação, o framing negativo deve ser usado para motivar ações preventivas.

No entanto, há controvérsia em relação ao uso de mensagens negativas ou com alto teor de incitação ao medo. Se for muito forte, o uso de apelo ao medo pode levar o consumidor a bloquear e a rejeitar a mensagem. Nesse sentido, a metodologia para manipulação do efeito framing usada neste trabalho deve ser uma alternativa viável e mais sutil. Essa forma de estruturação da mensagem exige o domínio de uma técnica não encontrada ou desenvolvida na criação das mensagens de marketing, de modo geral.

Como a exemplo da prática do marketing em serviços e produtos, as campanhas de saúde devem ser planejadas, testadas e medidas. É necessário também que sejam acompanhadas de ações concretas de educação e de assistência social, em vários níveis da sociedade, em certos casos, por um longo tempo. As campanhas de vacinação de determinadas doenças, por exemplo, já estão inseridas nos hábitos de grande parte da população brasileira. As vacinas mais comuns possuem alta eficácia percebida e um histórico importante de redução, ou mesmo erradicação, de doenças perigosas. Elas são quase uma 
unanimidade entre a comunidade médica, que educa a população sobre sua importância continuamente. Além disso, campanhas de vacinação são periódicas, recebem grande apoio social e logístico, o que garante distribuição intensiva e gratuita. A consistência das ações de comunicação e de distribuição, aliadas à eficácia do produto e à disponibilidade gratuita, são apenas parte do sucesso.

No Brasil, enfrentamos uma série de problemas sociais e econômicos que não podem ser subestimados. A deficiência na educação básica impede que grande parte da população compreenda mensagens aparentemente bem construídas. Existe ignorância em relação a problemas relacionados à saúde, falta de assistência e investimentos precários em políticas de prevenção. Muitas campanhas são elaboradas e aprovadas por pessoas que não possuem conhecimento profundo do público que as receberá, e interesses eleitorais provocam sérias distorções. Há desconhecimento das pesquisas sobre persuasão e falta de especialização nessa área. A medição de resultados não obedece a uma sistemática que possibilite a comparação entre campanhas diferentes ou entre campanhas sobre o mesmo assunto realizadas em diferentes épocas. Num país que, historicamente, investe pouco em saúde, o orçamento destinado a campanhas de prevenção é insuficiente e, muitas vezes, mal empregado. Mesmo iniciativas de organizações não governamentais e de empresas privadas carecem de planejamento e de conhecimento do assunto.

Espera-se que os resultados deste estudo tragam uma contribuição ao planejamento de campanhas de serviço público que objetivam provocar mudanças de comportamento. Pesquisas devotadas a tornar mensagens relacionadas à saúde mais efetivas prestam um importante serviço a vários tipos de organizações, como empresas, governos e organizações não governamentais. Em termos teóricos, há o enriquecimento do conhecimento sobre os mecanismos da mensagem publicitária e dos processos de julgamento e tomada de decisão do consumidor. Em termos práticos - considerados os grandes investimentos necessários em propaganda diante das limitações orçamentárias de governos e instituições - há o desenvolvimento de uma nova abordagem a ser considerada na formulação das mensagens de campanhas de utilidade pública.
Estudos adicionais no campo da comunicação de saúde podem estender esta pesquisa por meio da investigação mais detalhada dos efeitos moderadores de sentimentos, segmento social, grau de instrução, apenas para citar alguns. Devem também devem ser encontradas diferenças de percepção de medo ou de risco em diferentes culturas e em diferentes contextos sociais.

Neste estudo, as variáveis sexo e idade não tiveram influência diferenciada na persuasão. Entretanto, medidas adicionais precisam ser investigadas mais atentamente em futuras pesquisas. Uma limitação deste trabalho é seu aspecto experimental, uma vez que o ambiente controlado pode ter criado níveis artificiais de atenção para o assunto. Sua replicação em ambientes mais realistas e com outros materiais pode vir a se mostrar útil. Novos estudos de campo devem se utilizar de outros tipos de mídia e de mensagem, incluindo a utilização de imagens.

\section{Referências}

AAKER, D.; KUMAR, V.; DAY, G. Pesquisa de marketing. São Paulo: Atlas, 2001.

ARAÚJO, T.; GRAÇA, C.; ARAÚJO, E. Estresse ocupacional e saúde: contribuições do Modelo Demanda-Controle. Ciência \& Saúde Coletiva, Rio de Janeiro, v. 8, n. 4, p. 991-1003, 2003.

AVILA, M.; SÁ, I. Tomada de decisão: um teste da teoria dos prospectos. In: ENCONTRO NACIONAL DA ASSOCIAÇÃO NACIONAL DOS PROGRAMAS DE PÓS-GRADUAÇÃO EM ADMINISTRAÇÃO, 2o., 1996. Anais... Angra dos Reis: Anpad, 1996.

BALASSIANO, M.; RODRIGUES, E.; PIMENTA, R. Estresse ocupacional na administração pública Brasileira: uma análise baseada no modelo de relações estruturais. In: ENCONTRO NACIONAL DA ASSOCIAÇÃO NACIONAL DOS PROGRAMAS DE PÓS-GRADUAÇÃO EM ADMINISTRAÇÃO, 29., 2005. Anais... Brasília: Anpad, 2005.

BASTOS, N. et al. Qualidade de vida no trabalho e estresse ocupacional: uma pesquisa com professoras do curso de Pedagogia da Universidade de Fortaleza. In: ASSEMBLÉIA DO CONSELHO LATINO-AMERICANO DE ESCOLAS DE ADMINISTRAÇÃO (CLADEA), 37., 2002. Anais... Porto Alegre: CLADEA, 2002. 
BAZERMAN, M. Judgment in Managerial Decision Making. 6. ed. New York: John Wiley \& Sons, 2006.

BLOCK, L.; KELLER, P. When to accentuate the negative: the effects of perceived efficacy and message framing on intentions to perform a health-related behavior. Journal of Marketing Research, Chicago, v. 32, p. 192-203, may 1995. BOCHNER, S.; INSKO, C. A. Communicator discrepancy, source credibility, and opinion change. Journal of Personality and Social Psychology, Washington, DC ,v. 4, n. 6, p. 614-621, dec. 1966.

BRUNER II, G.; HENSEL, J. Marketing scales handbook: a compilation of multi-item measures. Chicago: American Marketing Association, 1996.

CHANDRAN, S.; MENON, G. When a day means more than a year: effects of temporal framing on judgments of health risk. Journal of Consumer Research, Gainesville, v. 31, p. 375-389, sept. 2004.

CHANG, C. Interactive effects of message framing, product perceived risk, and mood - the case of travel healthcare product advertising. Journal of Advertising Research, New York, v. 47, n. 1, p. 5165, mar. 2007.

CHUN-TUAN, C. Health-care product advertising: The influences of message framing and perceived product characteristics.Psychology \& Marketing, New Jersey, v. 24, n. 2, p. 143-169, feb. 2007.

FREIRE, K.; NIQUE, W. O papel do envolvimento com o produto e do comprometimento com a marca em diferentes fases da lealdade de clientes. In: ENCONTRO NACIONAL DA ASSOCIAÇÃO NACIONAL DOS PROGRAMAS DE PÓSGRADUAÇÃO EM ADMINISTRAÇÃO, 29., 2005. Anais... Brasília: Anpad, 2005.

GIFFORD, K.; BERNARD, J. Influencing consumer purchase likelihood of organic food. International Journal of Consumer Studies, Malden, MA, v. 30, n. 2, p. 155-163, mar. 2006

HUERTAS, M.; CAMPOMAR, M. Apelos da propaganda e atitude do consumidor sobre o produto: devem equiparar-se? In: ENCONTRO NACIONAL DA ASSOCIAÇÃO NACIONAL DOS PROGRAMAS DE PÓS-GRADUAÇÃO EM ADMINISTRAÇÃO, 30., 2006. Anais... Salvador: Anpad, 2006.
JEUNON, E. Valores e orientação de consumo: proposta e validação de um modelo integrativo. In: ENCONTRO NACIONAL DA ASSOCIAÇÃO NACIONAL DOS PROGRAMAS DE PÓSGRADUAÇÃO EM ADMINISTRAÇÃO, 29., 2005. Anais... Brasília: Anpad, 2005.

KAHNEMAN, D.; TVERSKY, A. Prospect theory: an analysis of decision under risk. Econometrica, Chicago, v. 47, p. 263-291, 1979.

KARDES, F. R. Consumer Behavior and Managerial Decision Making. 2. ed. New Jersey: Prentice Hall, 2002.

KIM, Y. The role of regulatory focus in message framing in antismoking advertisements for adolescents. Journal of Advertising, Athens, v. 35, n. 1, p. 143-151. spring 2006.

LIPP, M. Stress na atualidade: qualidade de vida na família e no trabalho. Disponível em: <http:// www.estresse.com.br/o6-Para_Ler/o6.01.15-stress_ na_atualidade.html> Acesso em: 31 jul. 2007.

MACKENZIE, S. B.; LUTZ, R. J. An empirical examination of the structural antecedents of attitudes toward the ad in an advertising pretesting context, Journal of Marketing, Chicago, v. 53, p. 48-65, apr. 1989.

MAHESWARAN, D.; MEYERS-LEVY, J. The influence on message framing and issue involvement. Journal of Marketing Research, Chicago, v. 27, p. 361-367, aug. 1990.

MEYEROWITZ, B.; CHAIKEN, S. The effect of message framing on breast self-examination attitudes, intentions and behavior. Journal of Personality and Social Psychology, Arlington, v. 52, n. 3, p. 500-510, mar. 1987.

MATOS, C.; VEIGA, R. The effects of negative publicity on consumer attitudes: a replication and extension. In: ENCONTRO DE MARKETING (EMA), 1., 2004. Anais... Porto Alegre: Anpad, 2004.

PALÁCIOS, M.; DUARTE, F.; CÂMARA, V. Trabalho e sofrimento psíquico de caixas de agências bancárias na cidade do Rio de Janeiro. Cadernos de Saúde Pública, Rio de Janeiro, v. 18, n. 3, p. 843851, maio/jun. 2002. 
PEREIRA, M. et al. O fenômeno do stress no trabalho sob a ótica sócio-construcionista: a produção de sentidos em uma organização militar. In: ENCONTRO NACIONAL DA ASSOCIAÇÃO NACIONAL DOS PROGRAMAS DE PÓSGRADUAÇÃO EM ADMINISTRAÇÃO, 30., 2006. Anais... Salvador: Anpad, 2006.

PETTY, R.; CACIOPPO, J.; SCHUMANN, D. Central and peripheral routes to advertising effectiveness: the moderating role of involvement, Journal of Consumer Research, Gainesville, v. 10, p.135-145, sept. 1983.

RAGANO, R. Understanding statistics in the behavioral sciences. 2. ed. St. Paul: West Publishing, 1986.

ROSENFIELD, J. Information as if understanding mattered, Fast Company, New York, n. 32, p. 209219, mar. 2000.

ROTHMAN, A. et al. The influence of message framing on intentions to perform health behaviors. Journal of Experimental Social Psychology, San Diego, v. 29, p. 408-433, 1993.

ROTHMAN, A. et al. The strategic use of gainand loss-framed messages to promote healthy behavior: how theory can inform practice. Journal of Communication, New York, v. 56, p. 202-220, sept. 2006.

SERPA, D.; AVILA, M. Percepção sobre preço e valor: um teste experimental. RAE Eletrônica, São Paulo, v. 3, n. 2, art. 13, p.1-19, jul./dez. 2004.

SIGNORELLI, N. Mass media images and impact on health: a source book. Westport: Greenwood Press, 1993, p.1-240.
SIMONSON, I. Get closer to your customers by understanding how they make choices, California Management Review, Berkeley, p. 68-84, summer 1993.

SMITH, G.; NAGLE, T. Frames of reference and buyer's perceptions of price and value. California Management Review, Berkeley, v. 38 n. 1, p. 118116, Fall 1995 .

SPARRENBERGER, F.; SANTOS, I.; LIMA, R. Associação de eventos de vida produtores de estresse e mal-estar psicológico: um estudo de base populacional. Cadernos de Saúde Pública, Rio de Janeiro, v. 20, n. 1, p. 249-258, jan./fev. 2004. SPARRENBERGER, F.; SANTOS, I.; LIMA, R. Epidemiologia do distress psicológico: estudo transversal de base populacional. Revista de Saúde Pública, Rio de Janeiro, v. 37, n. 4, p. 434439, 2003.

TANNER, J.; HUNT, J.; EPPRIGHT, D. R. The protection motivation model: a normative model of fear appeals. Journal of Marketing, Chicago, v. 55, p. 36-45, july 1991.

TVERSKY, A.; KAHNEMAN, D. The framing of decisions and the psychology of choice. Science Magazine, Washington, v. 211, n. 4481, p. 443-458, 30 jan. 1981.

TVERSKY, A.; KAHNEMAN, D. Choices, values and frames. American Psychologist, Washington, v. 39, n. 4, p. 341-350, 1984.

WINER, B. J.;BROWN, D. R.;MICHELS, K. M. Statistical principles in experimental design. 3. ed. Ohaio: McGraw-Hill Series in Psychology, 1991. 